\title{
2002 E. Mead Johnson Award for Research in Pediatrics Lecture: The Molecular Biology of the Anemia of Chronic Disease: A Hypothesis
}

\author{
CINDY N. ROY, DAVID A. WEINSTEIN, AND NANCY C. ANDREWS \\ Howard Hughes Medical Institute [N.C.A.]; Children's Hospital Boston [C.N.R., D.A.W., N.C.A.]; and \\ Harvard Medical School [C.N.R., D.A.W., N.C.A.], Boston, Massachusetts 02115, U.S.A.
}

\begin{abstract}
TBST
The anemia of chronic disease is a common disorder that
afflicts patients with a wide variety of inflammatory conditions
including arthritis, malignancies, infections, and inflammatory
bowel disease. It results in significant morbidity and may be
severe enough to require blood transfusions. The pathogenesis of
anemia of chronic disease is not fully understood, but poor
maintenance of red blood cell mass has been observed at three
levels: 1) iron is not efficiently recycled from reticuloendothelial
macrophages to erythroid precursors, 2) erythroid precursors
respond poorly to erythropoietin, and 3) red blood cell survival is
decreased. Whether each of these changes is related to the same
effector of the inflammatory process is unknown. We have had
the opportunity to investigate severe anemia of chronic disease in
an unusual group of patients with glycogen storage disease type
la. We found that anemia was directly related to the presence of
\end{abstract}
ACD, also called the anemia of chronic inflammation, is an acquired condition that affects patients with a variety of inflammatory disorders including infections, arthritis, inflammatory bowel disease, and cancer. ACD is the most common form of anemia among hospitalized and chronically ill patients. It is typically thought of as a mild to moderate decrease in red blood cell mass, but it can be severe enough to require transfusions.

Received August 21, 2002; acccepted August 26, 2002.

Correspondence: Nancy C. Andrews, M.D., Ph.D., Division of Hematology/Oncology, Enders 720, Children's Hospital Boston, 300 Longwood Avenue, Boston, MA 02115, U.S.A.; e-mail nandrews@enders.TCH.Harvard.edu

Support for this project was provided by a Clinical Research Center Grant from the Public Health Service, Division of Research Resources (NIH M01RR02172), a grant from the Association for Glycogen Storage Disease (U.S.A.), and the Children's Hospital Glycogen Storage Disease Research Fund. N.C.A. is an Associate Investigator of the Howard Hughes Medical Institute.

Dr. Andrews is the recipient of the 2002 E. Mead Johnson Award for Research in Pediatrics, presented at the 2002 Annual Meeting of the Pediatric Academic Societies, Baltimore, MD, U.S.A.

DOI: 10.1203/01.PDR.0000049513.67410.2D large hepatic adenomas that inappropriately produced a new peptide hormone, hepcidin. Hepcidin has recently been identified as part of the innate immune response and is a key regulator of cellular iron egress. Based on our findings in this patient group, we propose a central role for hepcidin in anemia of chronic disease, linking the inflammatory process with iron recycling and erythropoiesis. We present a hypothesis based on our findings.

(Pediatr Res 53: 507-512, 2003)
Abbreviations
ACD, the anemia of chronic disease
GSD1a, glycogen storage disease type 1a
IFN, interferon
TNF, tumor necrosis factor

This form of anemia often results in a significant, although subtle, decrease in quality of life and general well-being.

Ill patients can have anemia for a variety of reasons, including malnutrition, frequent phlebotomy, bleeding, myelosuppressive therapy, and others (1). The somewhat protean manifestations of $\mathrm{ACD}$, and the frequency with which it coexists with anemias of other types, can cause diagnostic confusion. ACD is often considered to be a diagnosis of exclusion. However, there are hallmarks that, in a compatible clinical setting, aid in establishing the diagnosis. ACD should be suspected when serum iron levels are low and the serum iron binding capacity (reflecting transferrin) is decreased, rather than elevated. These findings indicate that iron is being withheld from erythroid precursors, but the primary defect is not simple iron deficiency. Serum ferritin levels, often increased in inflammatory states, are usually within the normal range or elevated, again making the diagnosis of uncomplicated iron deficiency unlikely. Iron deficiency can be present in association with ACD, particularly when the patient had low iron 
stores to begin with, or when the inflammatory state has been present for many months. Accordingly, ACD is generally normocytic, but may be microcytic when the total body iron content is low. Examination of bone marrow macrophages usually shows adequate or increased storage iron, as the iron is retained and therefore unavailable for erythroid precursors. However, if iron deficiency is marked, or the inflammatory state has persisted for some time, macrophage iron may be inapparent (1).

At present, there is no single laboratory test that can definitively establish the diagnosis of ACD over iron-deficiency anemia. Some authors have proposed that the ratio of the serum transferrin receptor to the log of serum ferritin provides a useful index (2). It would be useful, however, to have a more straightforward test to make the diagnosis.

\section{PATHOGENESIS OF THE ANEMIA OF CHRONIC DISEASE}

The phenomenon of anemia in patients with inflammatory disorders has been appreciated since the early 1900s and much has been learned about the abnormalities associated with ACD. There are three observations that are highly characteristic. First, and perhaps most importantly, the normal flow of iron between tissues is altered in ACD. Short-term iron homeostasis can be thought of as a closed loop [Fig. 1, modified from (1)]. There is no regulated pathway for iron excretion through the liver and kidneys in humans; therefore, an inconsequentially small fraction of the body's iron endowment enters and leaves the system on a daily basis. Little exchange of iron occurs for purposes other than $\mathrm{Hb}$ production. Iron bound to transferrin is taken up by erythroid precursors and incorporated into $\mathrm{Hb}$. At the end of their lifespan (approximately 4 mo in normal individuals), senescent red blood cells are phagocytosed by specialized macrophages and the iron

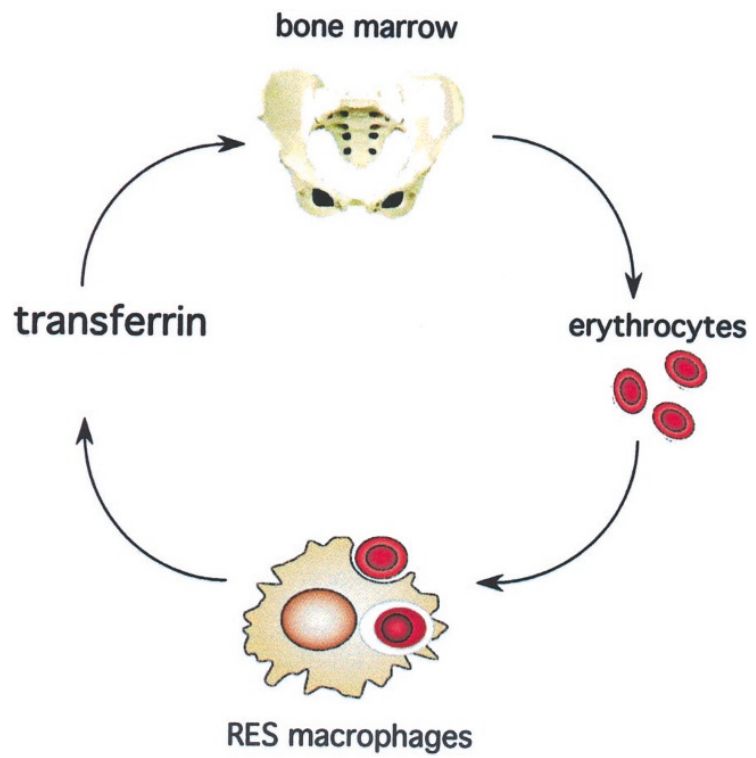

Figure 1. Iron utilization cycle. Short-term iron cycling can be described as a closed loop. The bulk of circulating iron is assimilated from transferrin in the plasma by developing red blood cells in the bone marrow. Mature red blood cells carry iron in $\mathrm{Hb}$. Senescent red blood cells are phagocytosed by macrophages of the reticuloendothelial system. Iron from heme is recycled to transferrin. There is no regulated mechanism of iron loss. from $\mathrm{Hb}$ is scavenged. A fraction of the iron is retained, but macrophages return the remaining iron to the plasma via transferrin, completing the loop. Erythropoiesis requires approximately $25 \mathrm{mg}$ of iron per day in an average adult, which must be provided by transferrin (1). ACD patients have significantly decreased transferrin saturations. Consequently, red blood cell mass decreases. Clinical efforts to elevate serum iron and improve erythropoiesis are often ineffective. Oral iron therapy is of no benefit, and i.v. iron therapy does not produce as vigorous a response as it does in patients with simple iron deficiency. The lack of response to iron is not caused by impaired erythroid acquisition of iron, because iron given in the form of diferric transferrin is rapidly taken up by erythroid precursor cells [reviewed in Cartwright (1)]. Taken together, these results suggest the transfer of iron from macrophages to transferrin within the closed loop is defective in ACD patients.

The second observation specific to ACD is that erythroid precursors from ACD patients respond poorly to erythropoietin, the growth factor necessary for red blood cell differentiation and $\mathrm{Hb}$ synthesis. Many investigators have described inflammation- or cytokine-induced changes in erythroid precursors that propagate this blunted erythropoietin response. Erythropoietin therapy yields variable responses in ACD patients. Often, a combination of iron and erythropoietin can be more effective than erythropoietin alone [reviewed in Means (3)]. This observation may be related to the changes in iron availability that occur in ACD. Hemoglobinization will not occur without sufficient iron, regardless of the presence of erythropoietin. In addition to the poor response of erythroid precursors to erythropoietin, individuals with ACD have also been observed to have inappropriate erythropoietin levels when the severity of their anemia is taken into account. Endogenous erythropoietin levels are usually increased in ACD, but not to the same extent as is seen in patients with similar degrees of anemia resulting from iron deficiency alone (3).

Finally, the observed lifespan of circulating red blood cells in individuals with ACD is decreased, apparently because they are more avidly phagocytosed by cells of the reticuloendothelial system (1). When normal red cells are given to a patient with $\mathrm{ACD}$, those cells are more rapidly cleared from the circulation. In a sense, the normal system that recovers iron from effete red cells is "revved up." The increased activity of reticuloendothelial macrophages in ACD may represent an attempt to increase the amount of iron available to developing red blood cells.

Whatever the mechanism, the closed loop finds equilibrium again with the net effect of reducing red blood cell mass in response to the restriction of iron available for erythropoiesis. This simple model is only useful for understanding ACD in the short term, however. When the inflammatory state persists for a prolonged period of months to years, the intestinal iron transport step must be considered. Minor blood loss and exfoliation of skin and mucosal cells normally results in loss of between $0.5-2 \mathrm{mg}$ of iron per day (more in menstruating women). When this loss is not balanced by intestinal absorption of the same amount, body iron stores are gradually depleted. ACD can result in disruption of dietary iron absorption (4). Over time, this can lead to iron deficiency, particularly in patients who have other reasons for low iron stores. Patients 
with combined iron deficiency and ACD are likely to be more anemic, and more likely to have microcytosis, than patients with ACD alone.

Although nonheme intestinal iron transport is now fairly well understood [reviewed in Andrews (5)], less is known about the part of that process that is interrupted in ACD. The absorptive enterocytes of the intestine take up substantial amounts of iron from the diet. Like macrophage iron handling, enterocytes retain some iron and export the rest across their basolateral membranes to enter the circulation. Iron that is retained within the cells cannot contribute to the body's overall iron economy because it is lost when the enterocytes senesce and are sloughed into the gut lumen. The machinery required for iron egress from macrophages and enterocytes is likely conserved. The only identified protein that has been shown to facilitate iron export is expressed in macrophages and enterocytes (6-8). If ACD induces iron retention in macrophages, it might also induce iron retention in enterocytes, reducing transfer of iron to the blood.

To summarize, the iron manifestations of ACD may be simply explained by decreased iron release from two types of cells: reticuloendothelial macrophages and absorptive enterocytes (Fig. 2). This concept supports a hypothesis that there is a normal mechanism for coordinately regulating release of iron from reticuloendothelial macrophages and enterocytes, and that this is altered in ACD. Also consistent with this notion is the fact that in hereditary hemochromatosis, a disorder that is the phenotypic inverse of ACD, increased iron absorption, systemic iron overload, increased serum iron, and macrophage iron depletion occur. This constellation of findings can be explained by a perturbation in iron trafficking in the opposite direction, i.e. increased basolateral transfer of enterocyte iron to the plasma, and increased release of iron from reticuloendothelial macrophages. In that case, the hypothesis would be
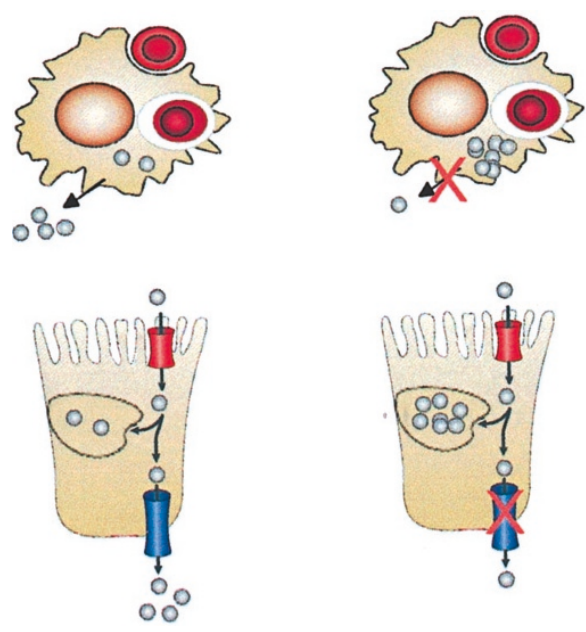

Figure 2. ACD is a defect in the release of iron from cells. The early hypoferremia and later iron deficiency observed in individuals with ACD can be explained by impairment of iron egress from macrophages and enterocytes. Low serum iron values and normocytic anemia are observed early and are most likely attributable to iron withholding by macrophages. If iron egress is impaired for weeks to months, the small contribution of dietary iron becomes apparent as iron stores in the liver and bone marrow macrophages also diminish and the anemia becomes microcytic. that the same regulatory system is perturbed, but in the opposite direction. This concept is shown in Figure 3.

\section{ACD IN PATIENTS WITH TYPE 1A GLYCOGEN STORAGE DISEASE}

Serendipitously, we encountered a group of patients who offered new insights into the postulated regulatory system, and how ACD might come about. GSD1a is an autosomal recessive disorder caused by deficiency of glucose-6-phosphatase, which catalyzes the terminal reactions of both glycogenolysis and gluconeogenesis. This disorder was lethal until it was discovered that continuous provision of glucose, through i.v. administration or frequent feedings of uncooked cornstarch, allowed patients to survive with a good quality of life. Effective therapies have been used for several decades now, allowing many patients to survive into adulthood. Unfortunately, although the metabolic manifestations of the disease are largely controlled, older survivors have developed a variety of complications, including hepatic adenomas and anemia (9).

We became interested in this group of patients when two observations were made - that the anemia in GSD1a survivors had the characteristics of severe ACD, and that those patients with the largest hepatic adenoma tumor burden had the most severe anemia. The experiments described below summarize our investigation of these tumors and the associated anemia. The results were recently published (9).

Initially, we thought it was important to determine whether this was, in fact, ACD or whether it had its own, unique etiology. Although this has not yet been answered definitively, we will argue that it is indistinguishable from ACD. First, patients with this disorder have evidence of abnormal iron homeostasis. They have very little iron in the circulation, with transferrin saturations less than $7 \%$. Erythropoiesis appears to be restricted by the paucity of iron; patients are microcytic and have elevated red blood cell distribution widths. However, this is not simple iron deficiency — total iron binding capacities are not elevated, ferritin levels are generally not less than $15 \mathrm{ng} / \mathrm{mL}$, and, importantly, the patients do not improve when they are given oral iron. They show a partial response to i.v. iron when it is given in large doses, but the response is sluggish and incomplete. These results are consistent with defects in both macrophage iron release and intestinal iron absorption, resulting in the physiology of ACD accompanied by iron deficiency caused by long-term impairment of dietary iron uptake. Although the condition seen in these patients is undoubtedly more severe than generally expected with $\mathrm{ACD}$, there is no other form of anemia that this resembles more closely.

Our second goal was to determine why the problem with iron homeostasis was associated with hepatic adenomas. Initially, one might have presumed that large adenomas and profound anemia were independent markers of disease severity. However, two patients had their adenomas removed surgically, and in both cases, the anemia resolved without blood transfusion, bone marrow modulators, or iron therapy. The first patient (patient A) had massive involvement of the liver with adenomas, and underwent liver transplantation. Postoperatively his anemia resolved (Table). However, it was possible that this was related to a general improvement in his health 
Hemochromatosis Anemia of chronic disease

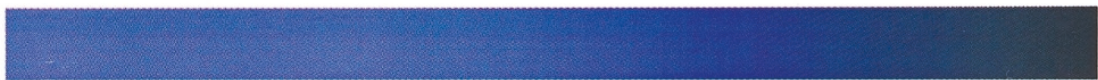

Too little:

Increased iron absorption

Increased macrophage iron release
Too much:

\section{Decreased iron absorption \\ Macrophage iron retention}

Figure 3. ACD and hemochromatosis are phenotypic opposites. Macrophage and enterocyte iron egress is implicated in the two diseases with opposite phenotypes. In ACD, impaired release of iron from macrophages decreases serum iron and impaired uptake of dietary iron eventually depletes iron stores. In hereditary hemochromatosis "leaky" macrophages and enterocytes allow too much iron into the system, eventually resulting in iron loading of parenchymal tissues. In this hypothetical schema, the bar represents the amount of circulating hepcidin, relative to what is appropriate for the iron status of the individual.

Table 1. Hematologic values for patients with GSDla before and after surgery that removed adenomas

\begin{tabular}{|c|c|c|c|c|c|c|c|}
\hline Patient & $\begin{array}{l}\text { Alk Phos } \\
\text { (U/L) }\end{array}$ & $\begin{array}{l}\text { Hct } \\
(\%)\end{array}$ & $\begin{array}{l}\mathrm{Hb} \\
(\mathrm{g} / \mathrm{dL})\end{array}$ & $\begin{array}{r}\mathrm{MCV} \\
(\mathrm{fL})\end{array}$ & $\begin{array}{l}\text { Serum Fe } \\
(\mu \mathrm{g} / \mathrm{dL})\end{array}$ & $\begin{array}{c}\mathrm{Ft} \\
(\mathrm{ng} / \mathrm{mL})\end{array}$ & $\begin{array}{c}\text { Adenoma hepc } \\
\text { (fold increase, } \mathrm{AU} \text { ) }\end{array}$ \\
\hline A (preop) & 280 & 24.2 & 6.9 & 62.5 & ND & ND & 5 \\
\hline $\mathrm{A}(+4 \mathrm{y})$ & 45 & 38.6 & 9.8 & 79.2 & ND & ND & \\
\hline $\mathrm{B}(+7 w \mathrm{w})$ & 46 & 36.6 & 12.3 & 83.7 & 34 & 6.6 & \\
\hline $\mathrm{B}(+5 \mathrm{mo})$ & 55 & 38.3 & 13.4 & 87.3 & 102 & 9.7 & \\
\hline $\mathrm{B}(+2 \mathrm{y})$ & 60 & 40.3 & 13.8 & 90.6 & 83 & 30 & \\
\hline
\end{tabular}

Both patients A and B presented with anemia [preoperatively (preop)] that improved after removal of adenomas by liver transplant (patient A, male, age 32 y) or resection (patient B, female, age $19 \mathrm{y}$ ). Red blood cell variables [hematocrit (Hct), Hb, and mean cell volume (MCV)]; alkaline phosphatase [(alk phos), a marker of disease severity]; and available iron all improved after removal of the adenomas [not determined (ND)]. The decrease in serum ferritin (Ft) observed immediately after surgery is likely related to renewed utilization of iron stores for erythropoiesis. Serum ferritin eventually returned to normal levels as iron stores were replenished. In situ hybridization (data not shown) verified inappropriate expression of hepcidin by adenoma hepatocytes when compared with hepatocytes from adjacent, unaffected liver [arbitrary units (AU)].

when his liver function was restored. The second patient (patient B) had a large, solitary adenoma at the tip of the left lobe of the liver, making the tumor resectable (in contrast to most GSD1a patients, who have unresectable adenomas). When her adenoma was removed, her anemia corrected within $7 \mathrm{wk}$, and her iron studies normalized (Table). As $6 \mathrm{wk}$ is the typical period needed for the red cell mass to recover after an erythropoietic block is relieved, it seemed highly likely that removal of the adenoma had, in effect, cured the anemia.

\section{GSD1A ADENOMAS PRODUCE A HORMONE THAT ALTERS IRON HOMEOSTASIS}

We next asked why the adenomas contributed to ACD. We considered the possibility that the tumors accumulated iron, sequestering it away from the erythron. However, staining of adenoma tissue and adjacent normal tissue with Perls Prussian blue showed no evidence of iron deposition.

Next we considered the possibility that the tumors were producing inflammatory cytokines that were responsible for causing ACD. The correlation between induction of TNF, IL-1, or IFNs and the development of ACD has been well established [reviewed in Means (3)]. Collectively, these cytokines have been postulated to decrease red cell survival, impair the response of bone marrow precursors to erythropoietin, and increase ferritin synthesis in reticuloendothelial macrophages. We compared gene expression in adenoma tissue versus adjacent unaffected liver tissue from patient $\mathrm{B}$ using a cDNA array containing sequences representing 180 cytokines and cytokine receptors. Neither the tumor nor the unaffected liver tissue produced appreciable amounts of TNF, IL-1, or IFN. There was a small ( $<2$-fold) increase in the expression of IL-13 and IL-16 in the adenoma, as well as a small increase in the expression of $\beta 2$-microglobulin, but none of these changes seemed likely to be related to the anemia caused by the adenomas.

At that point, a new candidate gene presented itself. Hepcidin is a small (20-25 amino acid) peptide hormone that is exclusively produced by the liver and induced by inflammation (10-13). Although initially identified because it acts as an antimicrobial peptide $(10,11,14)$, converging lines of experimental evidence strongly suggested a role for hepcidin in the regulation of iron homeostasis. First, impairment of murine hepcidin gene expression resulted in an iron overload phenotype similar to hereditary hemochromatosis (15). The increased liver iron and decreased splenic macrophage iron observed in these mice support the hypothesis that hepcidin normally acts to limit intestinal iron absorption and macrophage iron release. Second, loading of mice with carbonyl iron results in increased hepcidin expression (12), suggesting that it is part of a normal compensatory mechanism to decrease the amount of iron entering the body. Third, constitutive expression of a hepcidin transgene results in severe neonatal iron-deficiency anemia (13), indicating that hepcidin can completely (or nearly completely) shut down normal iron homeostasis. Because the features seen in our GSD1a patients with anemia resembled those expected from inappropriate expression of hepcidin, we 
considered the possibility that the adenomas, which are primarily made up of hepatocytes, might produce hepcidin.

We first needed to establish what levels of hepcidin expression to expect in our patients. Although we knew that hepcidin levels increased in iron overload (12), and that excess hepcidin expression caused iron-deficiency anemia (13), it had not yet been determined whether iron-restricted erythropoiesis led to decreased hepcidin production. Using mutant mouse strains that had iron-restricted erythropoiesis as a result of mutations disrupting iron transport, we showed that this was the case. Mice carrying mutations that disrupt intestinal iron transport (sla and $m k$ ) or iron uptake by erythroid precursors ( $m k$ and $h p x)$ are anemic $(5,16-19)$. Hepcidin expression was dramatically decreased in each of these mutant mice (Fig. 4). Whereas sla and $m k$ mice are anemic and iron deficient, $h p x$ mice are anemic, but iron loaded. Because hepcidin expression remains at low levels in $h p x$ mice despite such severe iron loading of the liver, we concluded that hepcidin must be primarily regulated by iron availability in the erythron. Therefore, we predicted that GSD1a patients with anemia should produce very little hepcidin, because they need to optimize intestinal iron absorption and macrophage iron recycling for erythropoiesis.

We next examined hepcidin mRNA expression by Northern blot analysis of three liver samples obtained from a healthy (iron replete) adult man, from patient B's unaffected liver, and from the adenoma. The healthy control sample showed robust

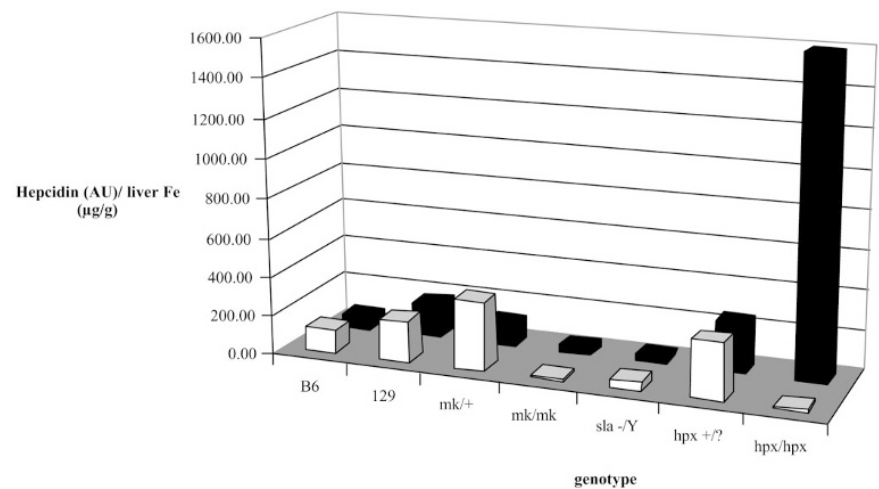

Figure 4. Hepcidin mRNA expression correlates with iron available for erythropoiesis rather than storage iron. This experiment is representative of multiple experiments with similar results. Hepcidin expression [white bars, arbitrary units $(A U)$ ] was quantified from Northern blot of mouse liver total RNA and compared with liver iron content expressed as micrograms per gram wet weight of liver tissue (black bars). Genotypes are given at the bottom: B6 $=\mathrm{C} 57 \mathrm{BL} / 6 \mathrm{~J}$ controls (low iron-loading strain), $129=129 \mathrm{SvEvTac}$ controls (high iron-loading strain), $m k /+=$ unaffected mice heterozygous for the $m k$ mutation, $m k / m k=$ affected $m k$ homozygotes, sla $/ \mathrm{Y}=$ affected sla male hemizygous mice on a C57BL/6J background, $h p x+/$ ? = unaffected littermates of $h p x$ mutant mice that may be either $+/+$ or $+/ h p x$ at the transferrin locus, $h p x / h p x=$ affected homozygous $h p x$ mutant mice. Mice with anemia as a result of defects in intestinal iron absorption $(\mathrm{mk} / \mathrm{mk}$ and $\mathrm{sla} / \mathrm{Y})$ or erythroid precursor iron uptake $(m k / m k$ and $h p x / h p x)$ turn off hepcidin expression when compared with the control mice. Hepcidin expression in wild-type B6 and 129 mice reflects the relative iron-loading phenotypes of those strains, and directly correlates with nonheme liver iron stores. However, $h p x / h p x$ mice have extensive iron loading of the liver, showing that liver iron stores do not correlate with hepcidin expression when anemia is present, and indicating the predominance of the erythroid regulator over the stores regulator in regard to hepcidin expression. hepcidin expression. Consistent with severe anemia, the unaffected liver tissue from patient B produced very little hepcidin. However, her adenoma tissue produced 10- to 30-fold more hepcidin than the unaffected liver. Although the adenoma produced an amount of hepcidin mRNA that was similar to that of the control, we knew from our experiments with anemic mice that this was inappropriately high expression.

To confirm that the adenoma hepatocytes were expressing hepcidin, we carried out in situ hybridization to detect hepcidin mRNA in tissue samples. We focused on the tumor margin, where we could compare hepcidin expression in the adenoma and unaffected liver tissue side by side. Consistent with our Northern blot results, we observed a striking difference between adenoma tissue, which expressed high levels of hepcidin mRNA, and normal tissue, which expressed very little in both patients (Table 1).

\section{DISCUSSION}

We presented evidence that inappropriate expression of hepcidin is associated with severe ACD in GSD1a survivors with large hepatic adenomas. However, a direct role for hepcidin in ACD associated with other inflammatory disorders has not yet been investigated. We believe that it is likely that hepcidin is a central mediator for ACD from diverse causes. It is a circulating hormone that is induced by inflammation, which blocks the release of iron from macrophages and interrupts intestinal iron absorption. These properties fit well with the iron-related manifestations of ACD.

Understanding the relationship between inflammation and hepcidin production will be important for determining which circumstances constitute "inappropriate" hepcidin expression. Details of the transcriptional and posttranscriptional regulation of hepcidin expression have not yet been worked out. It is possible that one or more of the inflammatory cytokines previously implicated in the pathogenesis of ACD triggers hepatic hepcidin production. This should be investigated. Importantly, levels of circulating hepcidin can be very tightly regulated through transcriptional mechanisms, because the molecule is very small, and is probably filtered into the urine by the kidneys, resulting in a relatively short half-life.

Although not all of the features of ACD may be fully attributed to hepcidin, it is likely that many features are associated with increased hepcidin expression. Certainly, its known effects on macrophages and intestinal absorptive cells can account for defects in iron recycling and intestinal iron absorption, respectively. Other features of ACD include decreased red cell lifespan and decreased responsiveness of erythroid precursors to erythropoietin, described above. Although these characteristics have been attributed to inflammatory cytokines, the involvement of hepcidin as a downstream signaling molecule should be investigated.

Why hepatic adenomas in GSD1a patients produce hepcidin inappropriately is not yet known, but our data suggest that the adenomas lack normal regulatory mechanisms that should turn off hepcidin gene expression. Identification of the signaling pathway that has turned on hepcidin expression in the first place may be important for understanding and treating ACD as 
a whole. Although it has not yet been demonstrated, we expect to find that hepcidin expression is increased in more conventional settings of ACD, such as rheumatoid arthritis, inflammatory bowel disease, cystic fibrosis, neoplasia, or chronic infection. Whether the path to induction of hepcidin is the same in each of these disorders remains to be determined.

Finally, the interdependence of antimicrobial and ironregulatory properties of hepcidin should be investigated. When ACD presents in association with autoimmune disorders, attenuating hepcidin expression may correct the anemia without complication. However, in association with neoplasia and chronic infection, the innate immune activity of hepcidin may be important for controlling the underlying disease. Antagonists that are specific for the target(s) of hepcidin on macrophages and enterocytes should be considered for treatment of ACD so that some iron is available to erythrocyte precursors, but innate immune activity is not compromised. Even still, such a treatment regimen might involve some risk as tumor growth and the proliferation of pathogens may be iron dependent.

\section{CONCLUSION}

We hypothesize that hepcidin plays a central role in the pathogenesis of ACD. This hypothesis will become testable when an antibody against hepcidin is available, and can be used to measure hepcidin levels in patient serum and urine. If we are correct, there is potential for producing drugs that target hepcidin or its as yet unknown receptor, to treat this very common form of anemia. Although not usually as severe as in our GSD1a patients, ACD undoubtedly impairs the quality of life of those affected; new therapeutic approaches would clearly be beneficial.

Acknowledgments. This review is based on the 2002 E. Mead Johnson Award Lecture, delivered at the 2002 Annual Meeting of the Pediatric Academic Societies on May 6, 2002. The original work described in this article was carried out by these authors in collaboration with Drs. Mark D. Fleming, Massimo F. Loda, and Joseph I. Wolfsdorf (9). We are especially grateful to Drs. John Crigler and Joseph Wolfsdorf for their enthusiasm and forethought when bringing this project together.

Note added in proof: Nemeth and colleagues (20) have recently reported increased levels of hepcidin in the urine of patients with anemia of inflammation. Their report broadens the spectrum of diseases that link hepcidin with ACD. The authors also report increased expression of hepcidin in hepatocytes cultured with interleukin-6, but not with IL-1 or TNF alpha. This observation establishes a direct connection between cytokine production and hepcidin expression.

\section{REFERENCES}

1. Cartwright GE 1966 The anemia of chronic disorders. Semin Hematol 3:351-375

2. Punnonen K, Irjala K, Rajamaki A 1997 Serum transferrin receptor and its ratio to ferritin in the diagnosis of iron deficiency. Blood 89:1052-1057

3. Means Jr RT 2000 The anaemia of infection. Baillieres Best Pract Res Clin Haematol 13:151-162

4. Cortell S, Conrad ME 1967 Effect of endotoxin on iron absorption. Am J Physiol 213:43-47

5. Andrews NC 2000 Iron homeostasis: insights from genetics and animal models. Nat Rev Genet 1:208-217

6. Donovan A, Brownlie A, Zhou Y, Shepard J, Pratt SJ, Moynihan J, Paw BH, Drejer A, Barut B, Zapata A, Law TC, Brugnara C, Lux SE, Pinkus GS, Pinkus JL, Kingsley PD, Palis J, Fleming MD, Andrews NC, Zon LI 2000 Positional cloning of zebrafish ferroportin1 identifies a conserved vertebrate iron exporter. Nature 403:776-781

7. McKie AT, Marciani P, Rolfs A, Brennan K, Wehr K, Barrow D, Miret S, Bomford A, Peters TJ, Farzaneh F, Hediger MA, Hentze MW, Simpson RJ 2000 A novel duodenal iron-regulated transporter, IREG1, implicated in the basolateral transfer of iron to the circulation. Mol Cell 5:299-309

8. Abboud S, Haile DJ 2000 A novel mammalian iron-regulated protein involved in intracellular iron metabolism. J Biol Chem 275:19906-19912

9. Weinstein DA, Roy CN, Fleming MD, Loda MF, Wolfsdorf JI, Andrews NC 2002 Inappropriate expression of hepcidin is associated with iron refractory anemia: implications for the anemia of chronic disease. Blood 100:3776-3781

10. Park CH, Valore EV, Waring AJ, Ganz T 2001 Hepcidin, a urinary antimicrobial peptide synthesized in the liver. J Biol Chem 276:7806-7810

11. Krause A, Neitz S, Magert HJ, Schulz A, Forssmann WG, Schulz-Knappe P, Adermann K 2000 LEAP-1, a novel highly disulfide-bonded human peptide, exhibits antimicrobial activity. FEBS Lett 480:147-150

12. Pigeon C, Ilyin G, Courselaud B, Leroyer P, Turlin B, Brissot P, Loreal O 2001 A new mouse liver-specific gene, encoding a protein homologous to human antimicrobial peptide hepcidin, is overexpressed during iron overload. J Biol Chem 276:78117819

13. Nicolas G, Bennoun M, Porteu A, Mativet S, Beaumont C, Grandchamp B, Sirito M, Sawadogo M, Kahn A, Vaulont S 2002 Severe iron deficiency anemia in transgenic mice expressing liver hepcidin. Proc Natl Acad Sci USA 99:4596-4601

14. Shike H, Lauth X, Westerman ME, Ostland VE, Carlberg JM, Van Olst JC, Shimizu C, Bulet P, Burns JC 2002 Bass hepcidin is a novel antimicrobial peptide induced by bacterial challenge. Eur J Biochem 269:2232-2237

15. Nicolas G, Bennoun M, Devaux I, Beaumont C, Grandchamp B, Kahn A, Vaulont S 2001 Lack of hepcidin gene expression and severe tissue iron overload in upstream stimulatory factor 2 (USF2) knockout mice. Proc Natl Acad Sci USA 98:8780-8785

16. Vulpe CD, Kuo YM, Murphy TL, Cowley L, Askwith C, Libina N, Gitschier J, Anderson GJ 1999 Hephaestin, a ceruloplasmin homologue implicated in intestinal iron transport, is defective in the sla mouse. Nat Genet 21:195-199

17. Fleming MD, Trenor CCI, Su MA, Foernzler D, Beier DR, Dietrich WF, Andrews NC 1997 Microcytic anemia mice have a mutation in Nramp2, a candidate iron transporter gene. Nat Genet 16:383-386

18. Fleming MD, Romano MA, Su MA, Garrick LM, Garrick MD, Andrews NC 1998 Nramp2 is mutated in the anemic Belgrade (b) rat: evidence of a role for Nramp2 in endosomal iron transport. Proc Natl Acad Sci USA 95:1148-1153

19. Trenor CC, Campagna DR, Sellers VM, Andrews NC, Fleming MD 2000 The molecular defect in hypotransferrinemic mice. Blood 96:1113-1118

20. Nemeth E, Valore EV, Territo M, Schiller G, Lichtenstein A, Ganz T 2002 Blood on-line prepublication. DOI:10.1182/blood-2002-10-3235 